\title{
Profil épidémiologique et clinique des cas suspects de COVID-19 reçus au CHU Yalgado Ouédraogo du Burkina Faso
}

\section{Epidemiological and clinical profile of suspected COVID-19 cases received at the Yalgado Ouédraogo Teaching Hospital in Burkina Faso}

Savadogo $\mathrm{M}^{1}$, Ouattara $\mathrm{A}^{2}$, Dahani $\mathrm{CK}^{2}$, Nikiéma $\mathrm{O}^{2}$, Traoré $\mathrm{S}^{2}$, Nagréongo $\mathrm{B}^{2}$, Sawadogo $\mathrm{N}^{3}$

1) Service des maladies infectieuses du CHU Yalgado Ouédraogo

2) Service des urgences médicales du CHU Yalgado Ouédraogo

3) Direction de la qualité du CHU Yalgado Ouédraogo

Auteur correspondant : Savadogo Mamoudou, Email : savadoma@gmail.com

\section{Résumé}

Introduction : la pandémie de COVID-19 n'a pas épargné le Burkina Faso qui a enregistré ses premiers cas le 09 mars 2020.

Objectifs : décrire le profil épidémiologique et clinique des cas suspects de COVID-19 au CHU Yalgado Ouédraogo de Ouagadougou

Patients et méthode : il s'agit d'une étude transversale conduite du 05 Avril au 04 Mai 2020 dans la zone d'accueil et de tri du CHU Yalgado Ouédraogo. Étaient inclus tous les patients suspects de COVID-19, conformément aux définitions de l'OMS.

\section{Résultats}

Sur les 2486 patients reçus au niveau de la zone d'accueil et de tri du CHU Yalgado Ouédraogo, 151 cas suspects de COVID-19 avaient été recensés, dont 14 cas confirmés, soit un taux de dépistage de 9\%. La majorité des patients $(71,5 \%)$ provenaient de la ville de Ouagadougou. L'âge moyen était de $53 \pm 18$ ans. Le sexe masculin prédominait (sex ratio $=1,25$ ). Cliniquement, $86 \%$ des patients étaient fébriles. Une toux était présente chez $69 \%$ des patients ; une dyspnée chez $60 \%$ avec une fréquence respiratoire moyenne de $29 \pm 10$ cycles/mn. La saturation pulsée en oxygène moyenne était à $86 \pm 13 \%$. La fréquence cardiaque moyenne était de $104 \pm 17 / \mathrm{mn}$. Une asthénie était notée chez $29 \%$; des céphalées chez 18\%; un mal de gorge chez 15,5\%; une douleur thoracique chez $11,5 \%$; une détresse respiratoire chez $11 \%$ des patients; un écoulement nasal et des nausées chez respectivement $7 \%$ des patients. Tous les cas suspects ont bénéficié de prélèvement nasopharyngé. La majorité des prélèvements $(76,2 \%)$ ont été effectués par l'équipe d'investigation de l'hôpital Yalgado. Les patients prélevés étaient hospitalisés dans $29 \%$ des cas. Six cas ont refusé le prélèvement. Neuf prélèvements ont été effectués en post mortem.

Conclusion : il est noté un polymorphisme clinique et un faible taux de dépistage de la COVID-19 chez les patients suspects, témoins d'un manque de spécificité des signes cliniques de la maladie.

Mots clés : cas suspects, clinique, COVID-19, épidémiologie.

\section{Abstract}

Introduction: The COVID-19 pandemic has not spared Burkina Faso, which recorded its first cases on March 9, 2020.

Objectives: Describe the epidemiological and clinical profile of suspected cases of COVID-19 at the Yalgado Ouédraogo Teaching Hospital in Ouagadougou.

Patients and approach: This is a descriptive cross-sectional study, from April 5, 2020 to May 4, 2020 in the reception and sorting area of the Yalgado Ouédraogo Teaching Hospital. Included were all suspect cases of COVID-19, in accordance with WHO definitions.

\section{Results}

Among 2,486 patients received in the reception and sorting area of the Yalgado Ouédraogo Teaching Hospital, 151 suspected cases of COVID-19 were identified, including 14 confirmed cases, for a screening rate of $9 \%$. The majority of patients $(71.5 \%)$ were from the city of Ouagadougou. The average age was $53 \pm 18$ years. Male sex predominated (sex ratio $=1.25$ ). Clinically, $86 \%$ of patients were febrile with a mean temperature of $38^{\circ} \mathrm{C} \pm 1.5$. Cough was present in $69 \%$ of patients; dyspnea in $60 \%$ with a mean respiratory rate of $29 \pm 10$ cycles $/ \mathrm{min}$. Mean pulsed oxygen saturation (Spo2) was $86 \pm 13 \%$. Mean heart rate was $104 \pm 17 / \mathrm{min}$. Asthenia was noted in $29 \%$; headache in $18 \%$; sore throat in $15.5 \%$; chest pain in $11.5 \%$; respiratory distress in $11 \%$ of patients; runny nose and nausea in $7 \%$ of patients respectively. All suspected cases were given nasopharyngeal swabs. The majority of the samples $(76.2 \%)$ were taken by the investigation team at Yalgado Hospital, hospitalized patients represented $20.9 \%$ of tested patients. Six cases of sample refusal were observed. Nine samples were taken postmortem.

Conclusion: A clinical polymorphism and a low detection rate of COVID-19 in suspect patients were noted, indicating a lack of specificity of the clinical signs of the disease.

Key words: COVID-19, epidemiology, clinical, suspect cases. 


\section{Introduction}

Les maladies infectieuses émergentes à potentiel épidémique constituent une menace permanente pour l'humanité. Dans le passé, l'humanité avait déjà subi des pandémies dévastatrices comme celles de la peste, de la grippe et du choléra. La pandémie à coronavirus, émergée en décembre 2019 en Chine, poursuit sa propagation à travers le monde malgré les mesures de lutte draconiennes entreprises par les Etats. Selon l'OMS, à la date du 03/09/2020 la maladie a tué officiellement, plus de 863570 personnes dans le monde dont plus de 29000 décès dans le continent africain. Le Burkina Faso à l'instar des autres pays est confronté à la COVID-19 depuis le 09 mars 2020. A la date du $1^{\mathrm{er}}$ Septembre 2020, le pays a enregistré 1386 cas de COVID-19 dont 55 décès (1). Face à cette situation, le ministère de la santé en collaboration avec ses partenaires techniques et financiers a élaboré un plan de riposte dont la mise en œuvre implique tous les acteurs du système de santé. A l'hôpital Yalgado Ouédraogo, une zone de tri des patients suspects de COVID-19 a été mise en place. L'objectif de notre étude est de décrire le profil épidémiologique et clinique des cas suspects de COVID-19 reçus à la zone de tri du CHU Yalgado Ouédraogo de Ouagadougou durant le premier mois de fonctionnement.

\section{Patients et méthode}

L'étude s'est déroulée à l'hôpital Yalgado Ouédraogo durant la pandémie. Les patients suspects ont été recrutés aussi bien de tri que dans les services d'hospitalisation. Il s'agit d'une étude transversale et descriptive durant la période allant du 05 Avril 2020 au 04 Mai 2020. Ont été inclus les patients présentant des signes suspects de la COVID-19 tels que définis par l'OMS :

Le cas suspect est défini comme tout patient présentant une infection respiratoire aigüe avec fièvre et au moins un signe respiratoire (toux, difficulté respiratoire), et des antécédents de voyage ou de séjour dans une localité ayant rapporté une transmission communautaire du COVID-19 dans les 14 jrs précédant le début des symptômes; ou tout patient présentant une infection respiratoire aigüe (IRA) avec fièvre et au moins un signe respiratoire (toux, difficulté respiratoire) et ayant été en contact avec un cas confirmé ou probable de COVID-19 dans les 14 jours précédant le début des symptômes; ou tout patient présentant une infection respiratoire aigüe sévère (IRAS) avec fièvre et au moins un signe respiratoire (toux, difficulté respiratoire) nécessitant une hospitalisation et devant l'absence d'une étiologie pouvant expliquer pleinement le tableau clinique.

Le cas probable est un cas suspect pour lequel le test au COVID-19 est indéterminé ou un cas suspect pour lequel le test ne pouvait pas être réalisé pour toute raison, ou les patients présentant des images évocatrices à l'imagerie.
Le cas confirmé est un cas suspect ou probable pour lequel la PCR au COVID-19 est positive.

Chaque patient suspect a bénéficié d'un interrogatoire et d'un examen physique complet avant la réalisation d'un prélèvement nasopharyngé. Des soins d'urgence sont administrés au besoin. Les prélèvements sont acheminés chaque jour au laboratoire de référence via le centre des opérations de réponse sanitaire (CORUS).

\section{Résultats}

En un mois d'activité au niveau de la zone d'accueil et de tri du CHU Yalgado Ouédraogo, un total de 2486 patients a été reçu. 151 cas suspects de COVID-19 ont été recensés dont 14 cas confirmés, soit un taux de dépistage de $9 \%$. La majorité des patients $(71,5 \%)$ provenaient de la ville de Ouagadougou. L'âge moyen des patients était de $53 \pm 18$ ans. Le sexe masculin prédominait ( $\operatorname{sex}$ ratio $=1,25)$. Les tableaux I présente les caractéristiques épidémiologiques des patients.

Tableau I : Répartition des patients selon les caractéristiques épidémiologiques

\begin{tabular}{|c|c|c|c|}
\hline \multicolumn{2}{|c|}{ Caractéristiques épidémiologiques } & \multirow{2}{*}{$\begin{array}{l}\mathrm{N} \\
61\end{array}$} & \multirow{2}{*}{$\begin{array}{c}\% \\
44,5\end{array}$} \\
\hline Sexe & Féminin & & \\
\hline & Masculin & 76 & 55,5 \\
\hline \multirow{2}{*}{$\begin{array}{l}\text { Age (Moyenne } \\
=53 \pm 18 \text { ans) }\end{array}$} & $\geq 60$ & 54 & 39 \\
\hline & $<60 *$ & 85 & 61 \\
\hline \multirow[t]{2}{*}{ Provenance } & Ouagadougou & 93 & 71,5 \\
\hline & $\begin{array}{l}\text { Hors } \\
\text { Ouagadougou }\end{array}$ & 37 & 28,5 \\
\hline \multirow[t]{3}{*}{ Prélèvements } & Sur patients & 136 & 90 \\
\hline & Post-mortem & 9 & 6 \\
\hline & Refusé & 6 & 4 \\
\hline \multirow{2}{*}{$\begin{array}{l}\text { Transfert en } \\
\text { centre de PEC }\end{array}$} & Accepté & 149 & 98,6 \\
\hline & Refusé & 2 & 1,4 \\
\hline \multirow{2}{*}{$\begin{array}{l}\text { Equipes de } \\
\text { prélèvement }\end{array}$} & CORUS & 36 & 24 \\
\hline & CHU YO & 115 & 76 \\
\hline \multirow{2}{*}{$\begin{array}{l}\text { Type } \\
\text { patients }\end{array}$} & hospitalisés & 30 & 20 \\
\hline & Externe & 121 & 80 \\
\hline
\end{tabular}

* dont 04 enfants

Sur le plan Clinique, $86 \%$ des patients étaient fébriles. Une toux était présente chez $69 \%$ des patients; une dyspnée chez $60 \%$ avec une fréquence respiratoire moyenne de $29 \pm 10$ cycles/mn. La saturation pulsée en oxygène moyenne était à $86 \pm 13 \%$. La fréquence cardiaque moyenne était de $104 \pm 17 / \mathrm{mn}$. Une asthénie était notée chez $29 \%$; des céphalées chez $18 \%$; un mal de gorge chez $15,5 \%$; une douleur thoracique chez $11,5 \%$; une détresse respiratoire chez $11 \%$ des patients ; un écoulement nasal et des nausées chez respectivement $7 \%$ des patients. . La majorité des prélèvements $(76,2 \%)$ ont été effectués par l'équipe d'investigation de l'hôpital Yalgado. Les patients prélevés étaient hospitalisés dans $29 \%$ des cas. Six 
cas ont refusé le prélèvement. Neuf prélèvements ont été effectués en post mortem. Les résultats du prélèvement et les caractéristiques cliniques des cas suspects figurent dans le tableau II.

Tableau II : Caractéristiques cliniques et para cliniques des patients suspects de COVID-19

\begin{tabular}{|c|c|c|c|}
\hline Signes & & $\mathbf{N}$ & $\%$ \\
\hline Fonctionnels & Fièvre & 125 & 86 \\
\hline & Toux & 99 & 69 \\
\hline & Céphalées & 26 & 18 \\
\hline & Asthénie & 41 & 29 \\
\hline & Dyspnée & 88 & 60 \\
\hline & Algie diffuse & 5 & 3,5 \\
\hline & Mal de gorge & 22 & 15,5 \\
\hline & Nausée & 9 & 7 \\
\hline & Vomissement & 6 & 4 \\
\hline & Rhinorrhée & 10 & 7 \\
\hline & Douleur poitrine & 16 & 11,5 \\
\hline & Hémoptysie & 3 & 2 \\
\hline Physiques & $\mathrm{FR}>20 \mathrm{c} / \mathrm{mn}$ & 25 & 66 \\
\hline & $\begin{array}{l}\text { Détresse } \\
\text { respiratoire }\end{array}$ & 15 & 11 \\
\hline & $\mathrm{FC}>90 / \mathrm{mn}$ & 18 & 78 \\
\hline & $\mathrm{T}^{\circ}>38,5^{\circ} \mathrm{C}$ & 51 & 57 \\
\hline & $\mathrm{SpO} 2 \geq 95 \%$ & 25 & 30 \\
\hline & $\mathrm{SpO} 2<95 \%$ & 39 & 61 \\
\hline & * Autres & 10 & 7 \\
\hline Résultats des & Positif & 14 & 9 \\
\hline prélèvements & Négatif & 137 & 91 \\
\hline $\begin{array}{l}\text { *Autres signes dor } \\
\text { égale à } 2 \%: \quad p \\
\text { anosmie, diarrh } \\
\text { fréquence resp }\end{array}$ & $\begin{array}{l}\text { la fréquence es } \\
\text { eur conjonctiv } \\
\text { anorexie, } \\
\text { toire; } \quad F C=\end{array}$ & $\begin{array}{l}\text { t infé } \\
\text { ale, }\end{array}$ & $\begin{array}{l}\text { eure ou } \\
\text { Fueusie, } \\
\text { FR= } \\
\text { quence }\end{array}$ \\
\hline $\begin{array}{l}\text { cardiaque } ; \quad S p O \\
\text { Oxygène } ; T^{\circ}=\text { temp }\end{array}$ & $\begin{array}{l}=\text { Saturation } \\
\text { rature }\end{array}$ & Part & lle en \\
\hline
\end{tabular}

\section{Discussion}

Débutée en Chine, l'infection à SARS-Cov-2 ou COVID-19, s'est rapidement propagée à travers le monde traduisant sa forte contagiosité comparativement à SARS-Cov-1 et au MERS-CoV qui avaient déjà été responsables d'épidémies plus limitées dans le temps et dans l'espace respectivement en 2002 et en $2012(2,3,4,5)$. Si les manifestations de la maladie sont dominées par des signes respiratoires, les connaissances actuelles lui attribuent d'autres manifestations cliniques. En effet la COVID-19 est aujourd'hui considérée comme une maladie pouvant toucher à la fois le cœur, les poumons, le tube digestif et le cerveau. Ainsi il a été décrit des accidents vasculaires et des embolies pulmonaires associés à la maladie. Sur le plan étiopathogénie, les nombreuses blessures au niveau des vaisseaux sanguins, occasionnées par le virus, déclencherait une cascade de coagulation qui affecte plusieurs organes expliquant l'atteinte multi-viscérale observée chez certains patients. Plusieurs études ont déjà documenté les effets délétères du virus sur les cellules endothéliales, avec comme conséquence des troubles de l'hémostase $(2,6,7)$. La variété des présentations cliniques de la maladie, et l'absence de spécificité des signes présentés par les patients, imposent un recours obligatoire à la $\mathrm{PCR}$ pour la confirmation du diagnostic $(8,9,10)$. L'âge moyen de nos patients était de $53 \pm 18$ ans, comparable à ce qui a été rapporté par plusieurs études chinoises $(4,5,8)$. Le sexe masculin était dominant dans notre étude tout comme dans les études de Chen, et de Wang en Chine $(11,12)$. Dans notre étude aucun cas confirmé de COVID-19 n'a été diagnostiqué chez les enfants, contrairement à l'étude de Chang $\mathrm{D}$, où la prévalence des cas pédiatriques était de $15 \%$ (13). Dans notre étude, la triade fièvre-toux-dyspnée dominait les manifestations cliniques observées. Nos résultats sont comparables à ceux de ChihCheng en Chine (14). La majorité de nos patients (61\%) présentaient à l'admission une saturation pulsée en oxygène inférieure à $95 \%$. Mikami et al retrouvaient les mêmes troubles mais chez des patients confirmés au COVID-19 (14).

\section{Conclusion}

Les patients suspects se recrutaient surtout chez des adultes jeunes de sexe masculin, résidant dans la capitale Ouagadougou. Les signes cliniques présentés par ces derniers étaient polymorphes. Beaucoup de cas suspects présentaient un tableau de sepsis sévère. D'où l'intérêt d'une unité de soins intensifs pour leur prise en charge. Il y avait un faible taux de dépistage de la COVID-19 chez les patients suspects, traduisant probablement une insuffisance de sensibilité des tests de dépistage. Il importe de renforcer l'équipement des laboratoires en moyens de diagnostic plus sophistiqués.

\section{Conflit d'intérêt : aucun}

\section{Références}

1) Ministère de la Santé Burkina Faso. Rapport de situation sur l'épidémie de la maladie à coronavirus (COVID-19) au Burkina Faso. 25 Août 2020.

2) Kuldeep Dhama, Sharun Khan, Ruchi Tiwari, Shubhankar Sircar, Sudipta Bhat, Yashpal Singh Malik Karam Pal Singh et al. Coronavirus Disease 2019-COVID-19. Clin Microbiol Rev. 2020 Oct; 33(4): e00028-20.

3) Yuxin Yan, Woo In Shin, Yoong Xin Pang, Yang Meng, Jianchen Lai, Chong You, et al. The First 75 Days of Novel Coronavirus (SARS-CoV-2) Outbreak: Recent Advances, Prevention, and Treatment. Int J Environ Res Public Health. 2020 Apr; 17(7): 2323.

4) Hossein Hozhabri, Francesca Piceci Sparascio, Hamidreza Sohrabi, Leila Mousavifar, René Roy, Daniela Scribano, Alessandro De Luca et al. The Global Emergency of Novel Coronavirus (SARSCoV-2) : An Update of the Current Status and Forecasting. Int J Environ Res Public Health. 2020 Aug; 17(16): 5648.

5) Meng-Yao Zhou et al. From SARS to COVID-19 : What we have learned about children infected with 
COVID-19. Int J Infect Dis. 2020 96:710-714. doi: 10.1016/j.ijid.2020.04.090

6) Bonny V, Maillard A., Mousseaux C., Plaçais L., Richier Q. COVID-19 : Physiopathologie d'une maladie à plusieurs visages. Revue de médecine interne 41(2020)375-389

7) Bonny V, Maillard A., Mousseaux C., Plaçais L., Richier Q. COVID-19 : Physiopathologie d'une maladie à plusieurs visages. Revue de médecine interne 41(2020)375-389

8) Rosanna W Peeling, Cathérine J Wedderburn, Patricia J Garcia, Debrah Baeras, Noah Fongwen, John Nkengasong, Amadou Sall, Amilcar Tanuri/serology testing in the COVID-19 pandemic response. Lancet infect Dis 2020

9) Esteban Ortiz-Prado, Katherine Simbaña-Rivera, Lenin Gómez- Barreno, Mario Rubio-Neira,b Linda P. Guaman, Nikolaos C Kyriakidis,Claire Muslin et al. Clinical, molecular, and epidemiological characterization of the SARS-CoV-2 virus and the Coronavirus Disease 2019 (COVID-19), a comprehensive literature review. Diagn Microbiol Infect Dis. 2020 Sep ; 98(1) : 115094.

10) Ioannis Kokkinakis, Kevin Selby , Bernard Favrat, Blaise Genton, Jacques Cornuz . Covid-19 diagnosis: clinical recommendations and performance of na-sopharyngeal swabPCR. Rev Med Suisse. 2020 16(689):699-701.

11) Chen N., Zhou M., Dong X., Qu J., Gong F., Han Y. Epidemiological and clinical characteristics of 99 cases of 2019 novel coronavirus pneumonia in Wuhan, China : a descriptive study. Lancet. 2020 ; $395: 507-13$

12) Wang D., Hu B., Hu C., Zhu F., Liu X., Zhang J. Clinical characteristics of 138 hospitalized patients with 2019 novel coronavirus-infected pneumonia in Wuhan, China. JAMA. 2020 ;323(11):1061-1069. doi:10.1001/jama.2020.1585

13) Chang D., Lin M., Wei L., Xie L., Zhu G., Dela Cruz C.S. Epidemiologic and clinical characteristics of novel coronavirus infections involving 13 patients outside Wuhan, China. JAMA. 2020 17;323(11):1092-1093. doi: 10.1001/jama.2020.1623.

14) Chih-Cheng Lai, Tzu-Ping Shih, Wen-Chien Ko, Hung-Jen Tang, and Po-Ren Hsueh. Severe acute respiratory syndrome coronavirus 2 (SARS-CoV-2) and coronavirus disease-2019 (COVID-19) : The epidemic and the challenges. Int $\mathbf{J}$ Antimicrob Agents. 2020 Mar ; 55(3) : 105924. 